\title{
Neural correlates of audio-visual modal interference inhibition investigated in children by ERP
}

\author{
WANG YiWen ${ }^{1,2}$, LIN ChongDe ${ }^{3 *}$, LIANG Jing ${ }^{1}$, WANG Yu ${ }^{1} \&$ ZHANG WenXin ${ }^{2 *}$ \\ ${ }^{1}$ Academy of Psychology and Behavior, Tianjin Normal University, Tianjin 300074, China; \\ ${ }^{2}$ School of Psychology, Shandong Normal University, Jinan 250014, China; \\ ${ }^{3}$ State Key Laboratory of Cognitive Neuroscience and Learning, Beijing Normal University, Beijing 100875, China
}

Received September 25, 2009; accepted December 15, 2009

\begin{abstract}
In order to detect cross-sectional age characteristics of cognitive neural mechanisms in audio-visual modal interference inhibition, event-related potentials (ERP) of 14 10-year-old children were recorded while performing the words interference task. In incongruent conditions, the participants were required to inhibit the audio interference words of the same category. The present findings provided the preliminary evidence of brain mechanism for the children's inhibition development in the specific childhood stage.
\end{abstract}

audio-visual modal, interference, inhibition, event-related potentials (ERP)

Citation: Wang Y W, Lin C D, Liang J, et al. Neural correlates of audio-visual modal interference inhibition investigated in children by ERP. Sci China Life Sci, 2011, 54: 194-200, doi: 10.1007/s11427-010-4127-9

In psychology, inhibition has been defined as an active process of suppression, excluding non-relevant information about tasks from working memory [1]. It is an executive function of the frontal cortex, which is related to social cognition and the theory of the mind [2,3]. Interference inhibition is one of the main types of inhibition processing which efficiently perceives and inhibits the interferential information in order to resolve the interference. In the Stroop task, the subjects were interfered with naming the print words' color by the meaning of the words in congruent conditions (such as a red "RED"), in incongruent conditions (such as a green "RED") and in neutral conditions (such as a red "XXX") [4]. The Stroop task is one of the main methods for studying interference inhibition. In the study of Mager et al. [5], when 30-year-old and 50-year-old adults were performing the Stroop task, a broad negativity developed after incongruent vs. congruent stimuli between 350 and $650 \mathrm{~ms}$. An age-related increase of the latency and amplitude of this

*Corresponding author (email: linchongde@263.net; zhangwenxin@sdnu.edu) negativity was observed. Markela-Lerenc et al. [6] found that N350-450 and P450-550 were greater in the incongruent as compared with the congruent and neutral conditions. Experimental materials may influence interferential effects of the Stroop task with regard to the processing course. In a study about the Stroop effect of Chinese characters [7], a greater negativity was found in the midline frontal-central scalp between 350 and $550 \mathrm{~ms}$ post-stimulus in incongruent conditions compared with congruent conditions. The results revealed that the difference between Chinese characters and English words had an influence on the temporal patterns of the Stroop interference effect. Liu et al. [8] found that the Stroop interferential effect primarily appeared between 300 and $500 \mathrm{~ms}$ post-stimulus, and in the latency of P330-390 in the ERP study of color judgment in the Stroop task concerning Chinese characters. Chen et al. [9] found that there were Stroop interferential effects in the reaction time (RT) of both Chinese and English materials, which were much stronger in Chinese tasks than in English. The different languages and different proficiencies had effects on the 
temporal patterns of Stroop interference effect.

The studies above were conducted on adults, but without comparable studies on children. Macleod [10] summarized 18 research results after reviewing Stroop research over half a century. He noted that the Stroop interferential effects were influenced by the age of the subjects and appeared in children at lower grades in primary schools, reaching the highest level at the second and third grade. As their reading ability developed, the interference in adulthood annually declined, and did not increase again until at nearly 60 years old. Hanauera et al. [11] found that there was a marked interferential effect in naming the picture while the words of the same category presented in the picture-word interferential task by children. However, this effect was not found in adults. Adults were more susceptible to the interference if the distracter presented and the pictures were in different categories, which showed the different characteristics of interferential effect at different ages.

Most studies about the interference effect were generally limited to the information of visual access. The evidence concerning the exploring interference of audio-visual information processing is particularly inadequate. One of the most commonly used paradigms in studying cross-modal interference is the picture-word interference paradigm which focuses on the interactions between the two types of information respectively conveyed in words and pictures. There are two types of word presentation in auditory and visual forms, and the experimental materials consist of pairs of pictures and words in which one is the target stimulus that participants are asked to respond to and the other one is the distraction that participants are asked to ignore. Previous studies $[12,13]$ showed that the RT of naming the pictures was shorter when the interference words and pictures were of different semantic categories. By contrast, the RT was longer when interference words and pictures were from the same semantic categories. Therefore, the interference effect of semantic association interference words is stronger than that from no semantic association interference words.

Event-related potential technology has been used to study the brain mechanism of the psychological development of children [14,15] for non-invasive and high time resolution characteristics. The inhibition capability is a key indicator for measuring the cognitive development of children. There are questions concerning the age characteristics and time-course of inhibition processing, and as to what the cognitive neuroscience mechanism of audio-visual cross-modal interference patterns. This study compares different ERP in words recognition under congruent conditions (the probe words and visual target words are the same) and incongruent conditions (the probe words are different from the visual target words, but the same as the auditory interference words) in the audio-visual cross-modal interference tasks of rendering words, as performed by children. It is assumed that the ERP components are greater in incongruent conditions than in congruent conditions in the present study, so as to explore the interference inhibition of auditory words in the same category with the visual target words recognized during the probe period. In this way, the interference inhibition of audio-visual cross-modal information in the brain processing of children would be revealed and cross-sectional characteristics at certain ages would also be provided.

\section{Materials and methods}

\subsection{Participants}

Fourteen 4th-grade pupils (six girls and eight boys, ranging from 10 to 11 years old, mean=10.2 \pm 0.4 ) participated in the experiment as paid volunteers. All participants were healthy, right-handed, and had normal or corrected to normal vision and hearing. Informed consent was obtained from all the participants' parents before the experiment.

\subsection{Stimulus materials}

The experimental materials were 120 pairs of double words which were from Chinese primary school textbooks within the range from the 1 st to the 8 th volume published by People's Education Press (2004 edition). All selected words were commonly used words in the Modern Chinese Frequency Dictionary [16]. The average word frequency and standard error of visual stimulus, auditory stimulus and probe stimulus are shown in Table 1 . There was no significant difference in word frequency, $F(2,359)=1.315, P=0.270$. Each pair of the double words was of the same category, such as "Bread-Cake", in which "bread" was in the visual form and "cake" was in the auditory form while the probe stimulus was "bread" or "cake" in the visual form.

The visual stimuli were shown in white text with Song typeface No. 42 on a black background in the center of the screen. The auditory stimuli were in standard mandarin with a female voice, processed with Cool Edit 2.1 recording into the stereo wav format of $-15 \mathrm{~dB}$. The accuracy of voice sampling was $44.1 \mathrm{kHz} \mathrm{s}^{-1}$ and the bit rate was 16 bits. Visual and auditory stimuli were both presented via a laptop.

\subsection{Experimental design and procedure}

The procedure of stimulus presentation in the audio-visual cross-channel interference task is shown in Figure 1. A

Table 1 Word frequency of visual and audio stimuli

\begin{tabular}{ccc}
\hline Stimulus & Amount & Frequency \\
\hline Visual words & 120 & $25.16 \pm 2.18$ \\
Audio words & 120 & $20.77 \pm 1.69$ \\
Probe words & 120 & $22.28 \pm 1.94$ \\
Total & 360 & $22.73 \pm 1.12$ \\
\hline
\end{tabular}



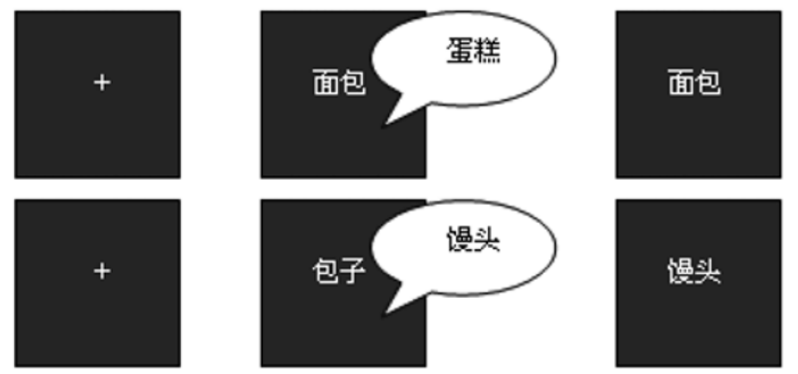

Audio-visual interference

Probe response

$500 \mathrm{~ms}$

$1000 \mathrm{~ms}$

$1500 \mathrm{~ms}$

Figure 1 Procedure of the audio-visual interference task.

cross-fixation was presented in the center of the screen for $500 \mathrm{~ms}$ first. A visual target word to be noticed and memorized by the subjects was shown on the screen, and an interference word of the same category was heard. Both words were shown for $1000 \mathrm{~ms}$. After that, the randomly changed inter stimulus interval (ISI) in the range of 400-600 ms was presented, for an average of $500 \mathrm{~ms}$. Finally, the probe word was presented for $1500 \mathrm{~ms}$ and the subjects were asked to judge "whether the word was the same as the one to be noticed and memorized". The subjects clicked the left mouse button if the probe word was the same as the visual target word (i.e., congruent conditions, no interference) or the right button if they were different (i.e., incongruent conditions, with interference). The responses of the left or right finger were counterbalanced between subjects to avoid the sequence effects.

The experiment was programmed with Stim2 software. Both the congruent conditions and incongruent conditions consisted of 60 trials with random variation of inter trails interval (ITI) among the trials within 400-600 ms, with an average of $500 \mathrm{~ms}$. Before the formal experiment started, the subjects would practice 20 similar exercise-trials to be familiar with the experimental procedure. In order to avoid the recognition effect, the stimulus materials in the practice were different from that in the formal experiment.

\subsection{ERP recording and analysis}

A portable ERP recording and analysis system (Neuroscan Inc. NuAmps) was used. The 40 channel electroencephalography (EEG) was recorded using a modified 10-20 system electrode cap referred to the bilateral mastoid reference and a forehead ground. The electrodes were placed outboard of both eyes to record the horizontal electro-oculography (EOG), and placed above and below the left eye to record the vertical EOG. All inter-electrode impedances were maintained below $5 \mathrm{k} \Omega$. EEG and EOG were amplified using a DC-100 Hz band pass and A/D sampling rate was $1000 \mathrm{~Hz} /$ channel.

The data was processed off-line after a continuous recording of EEG, in which the Neuroscan4.3 software was used in DC offset correction to correct vertical electrooculography (VEOG) and horizontal electro-oculography (HEOG) excluding the artifacts with an amplitude of over $\pm 100 \mu \mathrm{V}$. The EEG data of the probe response phase was analyzed in this study and the EEG of the correct response was overlaid. The epoch of ERP data analysis was $1500 \mathrm{~ms}$ after the probe stimulus while the baseline was $200 \mathrm{~ms}$ before the probe stimulus. The P1, N2, and N3 induced in the centrofront of the scalp in which the peaks were evident, were mainly analyzed in this study. The intervals of the peak amplitudes in $\mathrm{P} 1, \mathrm{~N} 2$ and $\mathrm{N} 3$ were respectively 150-250 ms (P200), 250-350 ms (N300) and 500-600 ms (N550). According to previous research and the purpose of this study, nine electrodes were selected, including Fz/F3/F4, FCz/FC3/FC4 and Cz/C3/C4. Three-way repeated measure analysis of variance (ANOVAs) was performed with the following three factors: 2 (tasks: congruent conditions vs. incongruent conditions) $\times 3$ (hemispheres: left hemisphere, midline, right hemisphere $) \times 3$ (electrodes). The $P$-values of the variance analysis were corrected with Greenhouse-Geisser correction.

\section{Results}

\subsection{Behavioral performance}

Accuracy and RT in congruent conditions and incongruent conditions are shown in Table 2. The differences of RTs in both conditions were significant in the paired sample $t$-tests, $t=-5.166, P<0.001$. The RT in incongruent conditions was significantly longer than that in congruent conditions, while the differences of the accuracies were not significant in both conditions.

\subsection{ERPs}

The grand average ERP waveforms evoked by the probe response in the frontal-central area are shown in Figures 2 and 3. Significant ERP components were induced in both congruent conditions and incongruent conditions at 200, 300 and 550 ms post-stimulus, which were named P200, $\mathrm{N} 300$ and N550. The respective latency of positive slow wave from 600 to $900 \mathrm{~ms}$ post-stimulus was basically consistent with the behavioral RT, which might be related to the choice and execution of the responses.

\subsubsection{P200}

Three-way repeated measure ANOVA analysis of the am-

Table 2 The accuracy and RT of two conditions

\begin{tabular}{ccc}
\hline & Accuracy $(\%)$ & RT $(\mathrm{ms})$ \\
\hline Incongruent & $94.17 \pm 6.59$ & $788.30 \pm 154.43$ \\
Congruent & $96.79 \pm 3.59$ & $685.01 \pm 156.76$ \\
\hline
\end{tabular}



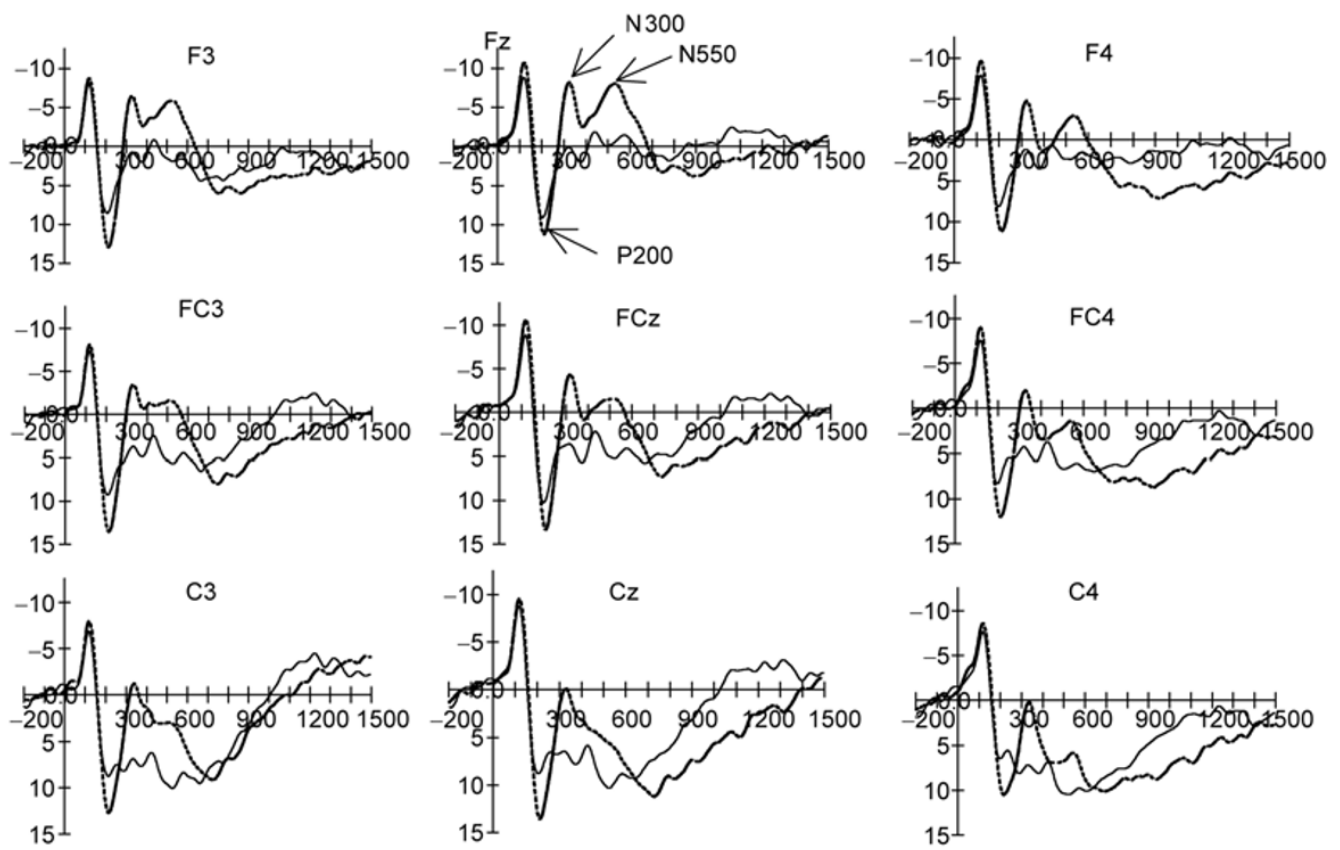

Congruent condition

Figure 2 Grand average ERP waveforms of two conditions $(n=14)$. Horizontal coordinate: stimulus presentation time (ms); vertical coordinate: ERP amplitudes $(\mu \mathrm{V})$.

plitudes of the P200 showed that the main effect of the tasks was significant, $F(1,13)=8.775, P<0.05$. The amplitudes in incongruent conditions $((13.235 \pm 1.963) \mu \mathrm{V})$ were significantly greater than those in congruent conditions $((10.469 \pm$ 2.194) $\mu \mathrm{V}$ ), while the main effects of hemispheres and electrodes were not significant. $F s=3.484 / 0.966, P>0.05$. In the interaction analysis, the interaction of the three factors task $\times$ hemisphere $\times$ electrode was significant, $F(4,52)=3.904$, $P<0.05$ in which the peak reached maximum $(14.647 \pm 2.080)$ $\mu \mathrm{V}$ at the $\mathrm{Cz}$ electrode in the midline brain in incongruent conditions while other interactions were not significant. It was found in the analysis of variance of the latency period of the P200 that the main effect of electrodes was significant, $F(2,26)=6.825, P<0.05$, and the latency period of $\mathrm{C} 3 / \mathrm{C} 4((214.119 \pm 4.779) \mathrm{ms})$ was the longest, significantly higher than $\mathrm{F} 3 / \mathrm{F} 4, \mathrm{FC} 3 / \mathrm{FC} 4$ with $P<0.05$. The interaction of the task $\times$ hemisphere was significant, $F(2,28)=6.062$, $P<0.05$. In the congruent conditions, the latency periods of the left hemisphere, the midline and the right hemisphere decreased in turn, while the latency periods of the right hemisphere, left hemisphere and the midline decreased in turn in incongruent conditions. The main effects and interaction of other factors were not significant.

\subsubsection{N300}

Three-way repeated measure ANOVA on the N300 amplitudes showed that the main effects of tasks, hemispheres and electrodes were all significant, $F s=12.917 / 4.636 / 38.398$,

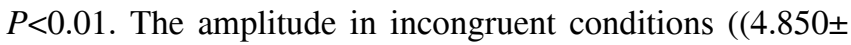

1.896) $\mu \mathrm{V}$ ) was significantly more negative than that in congruent conditions $((1.278 \pm 2.440) \mu \mathrm{V})$, and the amplitude at the midline $((2.781 \pm 2.137) \mu \mathrm{V})$ was also significantly more negative than that at the left hemisphere $((1.691 \pm 1.989) \mu \mathrm{V})$ and the right hemisphere $((0.886 \pm 2.003)$ $\mu \mathrm{V})$, in which the difference between the amplitudes at the midline and the right hemisphere was significant, $P<0.05$. The most negative amplitude was in F3/F4 ((4.598 \pm 1.912$)$ $\mu \mathrm{V}$ ) followed by $\mathrm{FC} 3 / \mathrm{FC} 4$ and $\mathrm{C} 3 / \mathrm{C} 4$ with a significant difference between each other, $P<0.05$, and all the interactions of three factors were not significant. The result of the three factor repeated measure analysis of variance on the latency period of the $\mathrm{N} 300$ showed that the main effect of the tasks was significant, $F(1,13)=9.810, P<0.008$. The latency period in incongruent conditions $((326.468 \pm 4.825)$ $\mathrm{ms)}$ was significantly longer than that in congruent conditions $((302.929 \pm 6.999) \mathrm{ms})$, and the interaction of task $x$ electrode was significant. The latency periods respectively descended from $\mathrm{C} 3 / \mathrm{C} 3$ to $\mathrm{FC} 3 / \mathrm{FC} 4$ to $\mathrm{F} 3 / \mathrm{F} 4$ in incongruent conditions and decreased from F3/F4, FC3/FC4 to $\mathrm{C} 3 / \mathrm{C} 3$ in congruent conditions. The main effects and interactions of other factors were not significant.

\subsubsection{N550}

For the amplitudes of N550, the main effect of tasks was extremely significant, $F(1,13)=10.531, P<0.01$. The amplitude in incongruent conditions $((1.812 \pm 1.580) \mu \mathrm{V})$ was significantly more negative than that in congruent conditions $((3.451 \pm 2.301) \mu \mathrm{V})$. The main effect of the electrodes was 
extremely significant, $F(2,26)=166.217, P<0.001$. The amplitude of negative measurements decreased from F3/F4 $((3.838 \pm 1.757) \mu \mathrm{V}), \mathrm{FC} 3 / \mathrm{FC} 4((0.804 \pm 1.844) \mu \mathrm{V})$, to $\mathrm{C} 3 /$ $\mathrm{C} 4((5.492 \pm 1.867) \mu \mathrm{V})$, in which the differences between each other was significant, $P<0.05$. Both the main effects and the interaction of the three factors in the hemispheres were not significant, and there was also no significant difference in the latency of N550.

\subsection{Topographic map}

The difference between the ERP waveforms evoked in incongruent conditions and congruent conditions appeared $200 \mathrm{~ms}$ post-stimulus which was found not only in the amplitude of P200 but also in the intensity of brain activation patterns in its topographic map analysis as shown in Figure 3. In incongruent conditions of P200 at about $200 \mathrm{~ms}$ post-stimulus, except for in the bilateral temporal regions, the suppression of other encephalic regions in the scalp was more active compared with that in congruent conditions. In incongruent conditions of $\mathrm{N} 300$, the front scalp region including the central area and part of the parietal lobe was in the state of suppression compared with that in congruent conditions, in which the suppression in the frontal lobe was even stronger. In incongruent conditions of N550, the suppression in the front scalp regions was stronger than that in congruent conditions, in which the suppression in the central regions was more readily apparent.

\section{Discussion}

\subsection{P200 and the perception of probe stimuli}

In order to study children's dynamic processing of audio-visual interference inhibition, the different brain electrical activities in congruent conditions and incongruent conditions were compared. There was interference in incongruent

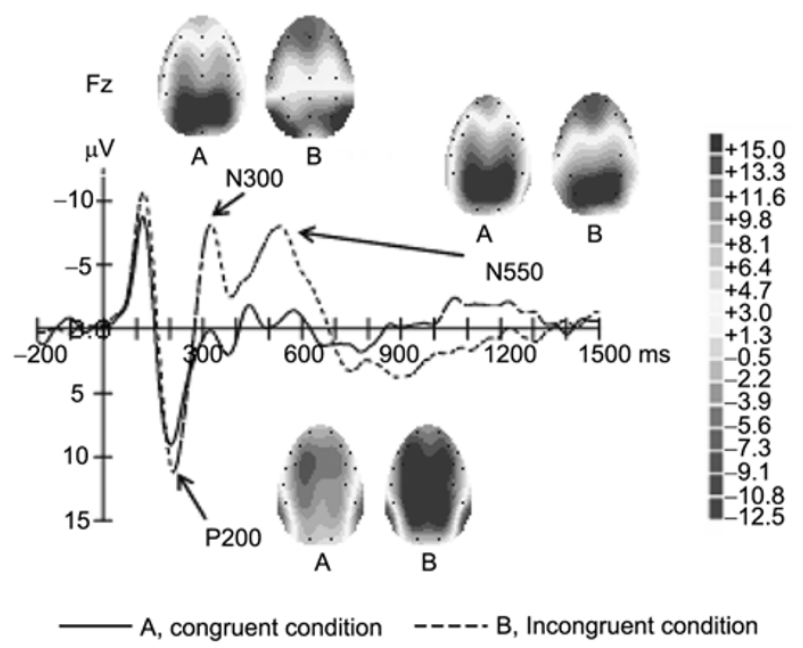

Figure 3 Topographic map in congruent and incongruent conditions. conditions causing the inhibition processing. The amplitude of P200 in congruent conditions was significantly smaller than that in incongruent conditions at about $200 \mathrm{~ms}$ after the probe stimulus was presented, indicating that the subjects may have initially apperceived the distinction of auditory interference words and visual target words matching the probe stimulus at the early stage when the stimulus was presented. In incongruent conditions, the electrical activities of the brain were influenced by interference stimuli when identifying the probe stimulus and became much stronger than that in congruent conditions.

The behavioral data of this study indicated that the RT was significantly longer in incongruent conditions than that in congruent conditions, reflecting the interference effects. The interference and target stimulus in the present study were of the same category, so the interference effects of children when reacting would be marked. Previous studies of the picture-word interference task also showed that when interference words and pictures were of the same semantic category, the RT of naming pictures was longer, which was the semantic interference effect. Yang et al. [17] studied the inhibition mechanism of external interference in processing visual Chinese vocabulary and sentences. It was found that the efficiency of the inhibition mechanism was influenced by the semantic relationship between interference materials and target materials. The closer the relationship was, the harder to inhibit the interference materials, which could be explained with the spreading activation model. In this study, the interference materials and target materials belonged to the same category with a closer relationship in the concept network, proposition network and the semantic space, with which the inferences were more easily activated. Therefore, the interference stimuli were difficult to be inhibited in processing the target materials [18]. According to the relative category level theory [19], the key factor which decided the degree of semantic effects was the relative category level of interference words and target words: If the two concepts were on the same level of the same category, there would be semantic interference. Otherwise, there would be semantic promotion. Therefore, the experimental results are explainable with the relative category level theory.

According to the topographic map, the brain electrical activity induced in congruence conditions is mainly distributed in the centro-frontal regions, while the distribution in the incongruence conditions was much broader, which was mostly distributed in the parietal-occipital regions in addition to the centro-frontal regions. Therefore, when the subjects were distinguishing the interference stimuli, they needed to activate more regions in brains, especially the parietal-occipital regions. Thus it was necessary for the subjects to concentrate on the features of information related to the task and inhibit the irrelevant interference information which required more cognitive resources. Under incongruent conditions, children mainly identified the stimuli and made a basic process by activating the parietal-occipital 
areas in order to optimize cognitive control [20].

\subsection{Time course of audio-visual interference inhibition}

It was found that in incongruent conditions auditory interference information of the same category needed to be inhibited, which induced significant ERP negativities of N300 and N550. These negativities were mainly distributed in the frontal lobe regions in which the amplitudes in incongruent conditions were greater than that in congruent conditions, decreasing from the prefrontal cortex to the central region. Seen from the topographic map of N300 and N550, negative patterns of activity were caused in incongruent conditions in the central regions, indicating that there was inhibition in the frontal lobe when the posterior scalp regions were activated and the most activated regions were the parietal-occipital regions. Compared with congruent conditions, the inhibition scope of the front scalp region was broader in incongruent conditions including the front area, central area and part of the parietal lobe. As the experiment proceeded, the inhibition scope in the front of the scalp became smaller from N300 to N550 and the activation of the posterior regions gradually increased, which showed that N300 and N550 might be ERP indicators of inhibition interference information in the frontal lobe region. The sustained activation of the posterior scalp might reflect children's development state of the prefrontal region, and the posterior scalp regions also needed to be involved in identifying and inhibiting the interference effect. In the study of the interference effect of adults, it was the frontal region that inhibited the interference effects. Markela-Lerenc et al. [6] found colors and meanings of words in incongruent conditions evoked a greater negativity in the $350-450 \mathrm{~ms}$ post-stimulus than that in congruent conditions in Stroop task. Source analysis of the difference wave (incongruent-congruent) indicated that a generator localized in the left prefrontal cortex contributed to this effect. Between $450-550 \mathrm{~ms}$ post-stimulus, a more positive wave would be evoked in incongruent conditions of which the generator was located in the right anterior cingulated cortex. Qiu et al. [7] studied event-related potentials of adult subjects in the Stroop task of Chinese characters and found that there was a greater negativity in the midline frontal-central scalp regions in incongruent conditions in the 350-550 ms post-stimulus presented compared with that in congruent conditions. The dipole source localization analysis of the wave which was obtained by subtracting the wave in congruent conditions from that in incongruent conditions indicated that the interference effects had a broader distribution in the prefrontal cortex. Chen et al. [9] investigated the dynamic changes of the Stroop interference effect in the epoch of brain in Chinese and English and found that the interference effect of Chinese characters was stronger than English words. The Chinese Stroop interference effects appeared between $350-550 \mathrm{~ms}$ post-stimulus with a greater negativity of ERP (N450) in incongruent conditions distrib- uted in the electrode in midline post-central scalp regions. In the Chinese Stroop study, the N450 component was found to contribute to the cognitive conflict and the selection of competing responses.

The N300 component in the centro-frontal regions at 250$350 \mathrm{~ms}$ post-stimulus in this study might reflect cognitive monitoring. Jonkman et al. [21] found that N300 was located on both sides of the medial frontal lobe (near the ACC) of both adults and children according to the analysis of dipole sources. A localization of posterior source was required for children, and the posterior source was located in the occipital-temporal area for 6-7 years old children and in the parietal lobe for 9-10 years old children. The N550 component in the time window of 500-600 ms post-stimulus might reflect the inhibitions of the interference stimulus which might be related to semantic activation [22]. It has been found in the existing research that the false belief task containing inhibition components induced late ERP negativities, of which the dipole source was located in the cingulated cortex [2]. Because the tasks in this experiment were developed from the picture-word interference task with experimental materials in Chinese and subjects were children, the results were mainly compared with that of the Chinese Stroop studies. Peng et al. [23] concluded that P300 and the amplitude of the slow wave at $400 \mathrm{~ms}$ post-stimulus in incongruent conditions were higher than that in congruent conditions and the color and the meaning of Chinese characters were different for the children in grade six in primary school. This conclusion was similar to the result of this study, but the polarity of the ERP, which was positive, was different from that in this study, which was negative. The difference might be due to the differences between Stroop tasks and the word interference task in the probe response phase of this study, with cross-modal involved. Rebai et al. [24] found that an ERP negativity N400 was evoked in Stroop tasks, which has both similarities and differences with this study. There was no comparison of development changes of different age groups of children in this study. Therefore, it needed to be further improved and approved. Liu et al. [25] utilized adolescents as subjects, with comparisons of wave differences that were obtained by subtracting the wave in congruent conditions from that in incongruent conditions when subjects from junior middle school, high school and college took the Chinese Stroop task. It was found that the P3 of junior middle school students was more positive than that of high school and undergraduate students, and there were different features in the scalp distribution among the three type of students above. Jongen and Jonkman [26] compared the ERP of the 6-7 years old group, 8-9 years old group, 10-12 years old group of children and groups of adults when taking the Stroop task. Although the processes of stimulus interference control seem to reach mature levels relatively early in childhood (6-7 years), the development of response interference control appears to continue into late adolescence as 10-12 year-olds remained more susceptible to er- 
rors of response interference than adults.

The topographical map showed that when children was inhibiting the audio-visual modal inference information of the same category, the ERP negativities began to appear mainly in the frontal lobe region, the central region and part of the parietal-occipital regions at about $300 \mathrm{~ms}$ post-stimulus. At about $500 \mathrm{~ms}$ post-stimulus, the negative components disappeared in the parietal regions while the negativities in the centro-frontal region were sustained. The interference inhibition of adults mainly involved the prefrontal lobe and anterior cingulated cortex while the parietal lobe primarily participated in children's inhibition which gradually disappeared, indicating that the inhibition of interference information in children needed the assistance of the parietal cortex besides the frontal lobe and the participation of the posterior regions to inhibit the interference. Bunge et al. [27] proposed that children aged 8-12 would activate the posterior regions, including the bilateral parietal, bilateral middle temporal and bilateral occipital cortices, in effective inhibition by using an event-related fMRI study. To assist the immature higher processing network including the prefrontal cortex, the lower processing system was restored. According to this conclusion, the parietal cortex was involved in the activation of learned stimulus-response (S-R) associations, while occipito-temporal regions were often involved in basic processing, such as stimulus identification. Elder children reactivated these $\mathrm{S}-\mathrm{R}$ associations in order to test the suitability of the response, while it was for fundamentally processing the stimulus for younger children in order to optimize cognitive control (conflict monitoring or response inhibition) [20], indicating that children's frontal lobe regions participated when inhibiting the interference, as well as the assistance of the posterior regions so as to compensate for the prefrontal cortex which was still weak in the function of inhibiting.

This work was supported by the National Natural Science Foundation of China (Grant Nos. 30807780 and 30700238).

1 Bjorklund D F. Children's thinking. 4th ed. Belmont: Wadsworth/ Thomson Learning, 2003. 119-140

2 Wang Y W, Liu Y, Gao Y X, et al. False belief reasoning in the brain: An ERP study. Sci China Ser C-Life Sci, 2008, 51: 72-79

3 Wang Y W, Lin C D. Frontal involvement to executive control: Load effects reflected by ERPs (in Chinese). Acta Psychol Sin, 2005, 37: $723-728$

4 Stroop J R. Studies of interference in serial verbal reactions. J Exp Psychol, 1935, 18: 643-662

5 Mager R, Bullinger A H, Brand S, et al. Age-related changes in cognitive conflict processing: An event- related potential study. Neuro- biol Aging, 2007, 28: 1925-1935

6 Markela-Lerenc J, Ille N, Kaiser S, et al. Prefrontal-cingulate activation during executive control: Which comes first? Cogn Brain Res, 2004, 3: 278-287

7 Qiu J, Luo Y J, Wang Q H, et al. Brain mechanism of Stroop interference effect in Chinese characters. Brain Res, 2006, 1072: 186-193

8 Liu C, Liu Z. An ERP study of Stroop effect and reverse Stroop effect in Chinese color-word (in Chinese). J Nanjing Normal Univ (Social Science), 2007, 53: 95-100

9 Chen X, Qiu J, Yuan H, et al. The brain mechanism of the Stroop interference effect in Chinese and English (in Chinese). Psychol Sci, 2007, 30: 529-534

10 Macleod C M. Half a century of research on the Stroop effect: An integrative review. Psychol Bull, 1991, 109: 163-203

11 Hanauera J B, Brooks P J. Contributions of response set and semantic relatedness to cross-modal Stroop-like picture-word interference in children and adults. J Exp Child Psychol, 2005, 90: 21-47

12 Lupker S J. The semantic nature of response competition in the picture-word interference task. Mem Cogn, 1979, 7: 485-495

13 La Heij W. Components of Stroop-like interference in picture naming. Mem Cogn, 1988, 16: 400-410

14 Wang Y W, Lin C D, Lu Z H. Research progress in developmental cognitive neuroscience (in Chinese). Prog Nat Sci, 2006, 16: 15301535

15 Wang Y W, Chen G H, Liu Y, et al. Application of the neuroimaging in development of psychology (in Chinese). Prog Nat Sci, 2007, 17: 603-608

16 Language Teaching Institute of Beijing Language College. Modern Chinese Frequency Dictionary (in Chinese). Beijing: Beijing Language College Press, 1986

17 Yang L X, Chen Y M, Zhou Z J. The inhibition mechanism of different skilled language comprehenders in self-paced lexical processing (in Chinese). Acta Psychol Sin, 2001, 33: 294-299

18 Collins A M, Loftus E F. A spreading-activation theory of semantic processing. Psychol Rev, 1975, 82: 407-428

19 Caramazza A, Costa A. The semantic interference effect in the picture-word interference paradigm: Does the response set matter? Cognition, 2000, 75: B51-64

20 Bunge S A, Hazeltine E, Scanlon M D, et al. Dissociable contributions of prefrontal and parietal cortices to response selection. Neuroimage, 2002, 17: 1562-1571

21 Jonkman L M. The development of preparation, conflict monitoring and inhibition from early childhood to young adulthood: A go/nogo ERP study. Brain Res, 2006, 1097: 181-193

22 Carter C S, Braver T S, Barch D M, et al. Anterior cingulate cortex, error detection, and the online monitoring of performance. Science, 1998, 280: 747-749

23 Peng D, Guo T, Wei J, et al. An ERP study on processing stages of children's Stroop effect. Sci Technol Engineer, 2004, 4: 84-88

24 Rebai M, Bernard C, Lannou J. The Stroop's test evokes a negative brain potential, the N400. Int J Neurosci, 1997, 91: 85-94

25 Liu Z, Liu C. The age differences of Stroop and reverse Stroop effect between adolescences and young adults: An ERP study (in Chinese). Psychol Explor, 2007, 27: 48-54

26 Jongen E M, Jonkman L M. The developmental pattern of stimulus and response interference in a color-object Stroop task: An ERP study. BMC Neurosci, 2008, 9: 82

27 Bunge S A, Dudukovic N M, Thomason M E, et al. Immature frontal lobe contributions to cognitive control in children: Evidence from fMRI. Neuron, 2002, 33: 301-311

Open Access This article is distributed under the terms of the Creative Commons Attribution License which permits any use, distribution, and reproduction in any medium, provided the original author(s) and source are credited. 